\title{
Days of Molecular Medicine 2004
}

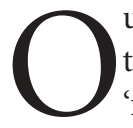

ur understanding of food intake and body weight regulation has come a long way since the 1951 discovery of a 'feeding center' in the rat hypothalamus. The early concept of a regulatory system with only two states-hunger and satiety-has given way to a much more sophisticated model of food intake, which has been largely fueled by the identification of the molecules that regulate this basic biological activity.

Recognizing the remarkable progress in this area of research, this year's Days of Molecular Medicine meeting focused on "Integrative Physiology and Human Diseases: Neurohormonal and Metabolic Pathways". The meeting, sponsored by Nature Medicine, the Institute of Molecular Medicine (UCSD) and The Wellcome Trust, took place at the Wellcome Trust Sanger Institute in England. Its broad topic reflected the fact that the regulation of food intake encompasses not only its neural control, but also the complex interactions between brain, gut, adipose tissue, liver and muscle.

The 1994 discovery of leptin as a fat-derived key regulator of feeding behavior with direct actions on the brain was a turning point in this field. It was therefore entirely appropriate for Jeffrey Friedman, discoverer of leptin, to deliver the keynote address, during which he reviewed seminal developments in the study of the regulation of food intake over the past decade. His talk covered four areas that have seen the most significant progress: the identification of signaling molecules that regulate food intake, the tight relationship between caloric intake and expenditure, the intricate interplay between fat, brain and other organs, and the discovery of mutations that lead to morbid obesity. This overview set the stage for the rest of the meeting, during which each of those areas received additional attention.

The number of molecules known to regulate feeding has increased significantly since the discovery of leptin. This growth has led to the mapping of intercellular pathways involved in the orexigenic or anorexigenic effects of molecules such as ghrelin, melanin-concentrating hormone and the derivatives of proopiomelanocortin (as discussed at the meeting by Roger Cone, David Cummings, Steve Bloom and other leading figures in the field). These talks provided an emerging view of the interactions between those pathways, and about the way in which organs such as the gut and adipose tissue communicate with the brain.

The study of the intracellular mechanisms that regulate food intake has also experienced remarkable progress. Presentations by Jeffrey Flier, Bruce Spiegelman, Barbara Kahn and others highlighted the fact that, although these intracellular signaling molecules are present in places as diverse as fat, liver and muscle, their actions are not the same in every organ, increasing even further the difficulty involved in understanding the control of metabolism.

Another recurrent theme of the meeting was the tight relationship between food intake and energy expenditure; normally, any reduction in caloric intake is matched by a proportional reduction of calorie utilization. Discovering ways to disentangle this relationship is a key challenge before finding new treatments for obesity, the prominence of which is on the rise. According to recent data from the World Health Organization (WHO), there are $~ 1$ billion overweight adults worldwide, 300 million of whom are obese. As excess body weight is a risk factor for cardiovascular disease, diabetes and other disorders, there is increasing awareness of obesity as a global health problem.

Despite this increasing awareness, the link between caloric intake and expenditure means that the WHO's recommendation to manage obesity through a healthy diet might not be sufficient, and alternative strategies might be required. In this regard, the identification of morbidly obese people with mutations in anorexigenic molecules provides an instructive lesson. The effectiveness of replacement therapy in some of these subjects (discussed at the meeting by Steve O'Rahilly) is an example of the potential for the development of anti-obesity therapeutic agents. Indeed, molecules with anorexigenic effects such as cilliary neurotrophic factor are already in clinical trials, as mentioned at the meeting by Mark Sleeman. And, as discussed by David Moller, other molecules such as melanocortin-4 receptor are being actively pursued as new targets for appetite suppressants. Whether any of these anti-obesity therapies turns out to have real beneficial effects remains to be demonstrated.

As in years past, the meeting offered an opportunity to honor individuals who have made exceptional contributions to mentoring and to public service. This year, the Mentorship Award was presented to Donald Metcalf for fostering the careers of many scientists and physicians in Australia. The Service Award was given to Bernard Barataud for his pioneering efforts in the development of Telethon and the Association française contre les myopathies, a leading European research foundation.

In 2005, Days of Molecular Medicine will return to the US. Together with the Institute of Molecular Medicine and the Salk Institute, we will collaborate on a meeting devoted to progenitor cells and regenerative medicine-another field that has seen explosive growth and that exemplifies the philosophy of the series: the translation of basic research into clinical applications. We hope you join us next year in San Diego. 